\title{
Entropy, Decoherence and Spacetime Splitting
}

\author{
Rodolfo A. Fiorini \\ Politecnico di Milano, Department of Electronics, Information and Bioengineering (DEIB), Milano, \\ Italy; E-Mail: rodolfo.fiorini@polimi.it; Tel.: +039-02-2399-3350; Fax: +039-02-2399-3360.
}

Published:

\begin{abstract}
The "Science 2.0" paradigm has not yet been completely grasped by many contemporary scientific disciplines and current researchers, so that not all the implications of this big change have been realized hitherto, even less their related, vital applications. Thus, one of the key questions in understanding the quantum-classical transition is what happens to the superpositions as you go up that atoms-to-apple scale. Exactly when and how does "both/and" become "either/or"? As an example, we present and discuss the observer space-time splitting case. In other words, we discuss spacetime mapping to classical system additive representation with entropy generation. We argue that it is exactly at this point that "both/and" becomes "either/or" representation by usual Science 1.0 approach. CICT new awareness of a discrete HG (hyperbolic geometry) subspace (reciprocal space) of coded heterogeneous hyperbolic structures, underlying the familiar $\boldsymbol{Q}$ Euclidean (direct space) surface representation can open the way to holographic information geometry (HIG) to recover system lost coherence and to overall system entropy minimization. CICT can help us to develop strategies to gather much more reliable experimental information from single experimentation and to keep overall system information coherence. This paper is a relevant contribution towards an effective and convenient "Science 2.0" universal framework to develop more effective physics modeling and simulation for innovative application and beyond.
\end{abstract}

Keywords: space-time split; quantum-classical transition; CICT; decoherence; entropy; statistical mechanics; combinatorial optimization; reversibility; quantum mechanics.

PACS Codes: 03.67.-a; 03.67.Ac; 03.67.Bg; 03.67.Hk; 03.67.Pp. 


\section{Introduction}

Science evolves through the continuous formulation of new hypotheses. Models are sound tools to investigate hypotheses and to check for specific predictions that have to be verified experimentally. The comparison of model predictions with experimental data allow both to refine the hypotheses and to develop more accurate models. Sometimes models that can even shed light on completely different phenomena. Models are even quite helpful in developing logical hierarchical structures. The great potential of nano-bio-technology is based also on the ability to deal with complex hierarchically structured systems from the macroscale to the nanoscale; this requires novel theoretical approaches and the competence to create models able to explain the dynamics at this scale. Geometric and analytical approaches seem to be very promising in all scientific areas, like for the study of brain processes. The comprehension and adaptiveness bring to a change of the internal brain parameters, in order to mimic the external transformation by the appropriate use of sensors and effectors. The current dynamics modeling of substances in the brain is still based on classic transport models; the improvement of them is a mandatory step for the deep comprehension of the brain functioning. For explaining the nanoscale phenomena, a deep understanding of electromagnetic (e.m.) and photonic interactions at this size scale is desirable, through experiments, theory and mathematical modeling $[1,2]$. An advance in system modeling, working from sub-pico to macro-level, is able to adequately study the nano-dynamics in the brain and is giving interesting ideas for future developments [3],[4]. Research on brain plasticity and circuitry also indicates that the brain is always learning, in both formal and informal contexts. The learning experiences produce a "chain action" of signaling among a group of neurons in some areas of the brain, with the modification of connections among neurons in determined areas of the brain and a consequent reorganization of these areas. In full accordance to Andrews and Weiss [5], chemical communication and key bio-molecular interactions in the brain occur at the nanoscale, therefore the idea of taking advantages of nanoscience for advances in the study of the brain structure and function is increasing in interest. Current experimental brain nanotechniques are developed by three complementary approaches [5]:

1) snapshots of connections in the brain by making thin physical slices and stacking electron microscopy images of these slices. This technique does not provide dynamic and chemical information;

2) dynamic voltage map of the brain, dealing with the brain as close relative of a computer $[6],[7],[8]$, with the aim to make accessible the emergent properties underlying the use and storage of information by mapping network activity versus single or small numbers of multiple unit recordings currently available;

3) the attempt to obtain functional chemical maps of the neurotransmitter systems in the brain, for investigating the genetic and chemical heterogeneity of the brain and the interactions between various neurotransmitter systems.

In all cases, the length scale ranges from the centimeter scale $(\mathrm{cm})$ (in mapping brain regions and networks), to the micrometer scale $(\mu \mathrm{m})$ (cells and local connectivity), to the nanometer scale $(\mathrm{nm})$ 
(synapses), to the single-molecule scale [5]. The current ability to perform neurochemical and electrophysiological measurements needs to be miniaturized, sped up, and multiplexed. Electrical measurements at time scales of milliseconds are uncomplicated, but getting to the nanometer scale and making tens of thousands measurements simultaneously in vivo remains arduous. Obtaining dynamic chemical maps at these scales is an even bigger challenge, so as problems in analysis, interpretation and visualization of data [5]. In two different papers we already discussed the major intrinsic limitations of "Science 1.0" arbitrary multiscale (AMS) modeling and strategies to get better simulation result by "Science 2.0" approach [9],[10]. We know that nonlinear differential equations, stiff and nonlinear dynamical systems, developed under the continuum hypothesis, are computed inefficiently on a digital computer. The IBM Blue Gene project is a simple example: it uses 4096 CPUs and 1000 Terabytes RAM, which, to simulate the mouse cortex requires $8 \times 10^{6}$ neurons, $2 \mathrm{x}$ $10^{1 \circ}$ synapses, $10^{9} \mathrm{~Hz}$ clock, 40 Kilowatts on digital support. The human brain uses about 85 billion neurons and estimated 100 trillion $\left(10^{14}\right)$ synapses [11], $10 \mathrm{~Hz}$ clock, and 20 watts [12] on biological support. This fact suggests the utility of going back to models of natural computation and therefore of looking at the Turing computation as a "coarse grain" of processes which are best suited to be described with geometric manifolds [13], [14],[15,],[16],[17],[18]. During the first phase of the development of quantum theory (1913-1927) three fundamental questions were posed:

1) the quantization of material motion, represented by discrete electron orbits;

2) the quantization of the field, in terms of the hypothesis of the emission and absorption of photons; and

3) the discontinuity of motion, represented by quantum jumps between stationary orbits.

With regard to points 1) and 2), the subsequent definition of quantum formalism led to quantum mechanics (QM) and quantum field theory (QFT), or, in other words, respectively to first and second quantization. It has been noted that QM and QFT still do not constitute an organic structure; waveparticle duality in particular, which proved so useful for the description of Einstein-Podolsky-RosenBell phenomena, has no place in descriptions based strictly on QFT [19]. The point 3) did not bring any particular development; it is still the source of a periodically renewing debate, in particular about the questions related to the wavefunction collapse (the anomalous postulate of quantum mechanics) and its incompatibility with special relativity [19]. In his classical book on quantum mechanical principles [20], Heisenberg delineates with his usual clarity two possible ways to build QM, both of which were coherent with the uncertainty principle:

A) a space-time description tending to save the classical concepts by means of a statistical connection;

B) adopting a pre-spatial mathematical scheme and a different concept of causality.

In the 1930 s there was no cogent reason to choose the second way. Nowadays, quantum nonlocality offers a valid reason to explore a different approach where the nonlocal features of quantum mechanics 
are not necessarily restricted to the entangled amplitudes, but are instead connected to a time-reversal approach [21], or some timeless background.

QFT has emerged from major paradigm shift with respect to Classical Physics which still provides the framework of the vision of nature of most scientists. This change of paradigm has not yet been completely grasped by many contemporary scientific disciplines and current researchers, so that not all the implications of this big change have been realized hitherto, even less their related, vital applications. So, the discreteness approach, developed under the QT (quantum theory) "discreteness hypothesis" assumption, has been considered in peculiar application areas only. It has been further slowly developed by a few specialists and less understood by a wider audience. It leads to the fresh QFT approach. According to Ball [22], following the classic explanation, to understand what the quantum-classical transition really means, consider that our familiar, classical world is an "either/or" kind of place. A compass needle cannot point both north and south at the same time. The quantum world, by contrast, is "both/and" and a magnetic atom model has no trouble at pointing both directions at once. The same is true for other properties such as energy, speed, etc. Generally speaking, they can take on a range of values simultaneously, so that all you can say is that this value has that probability. When that is the case, physicists say that a quantum object is in a "superposition" of states. Human worldview is about incompleteness: incompleteness of understanding, representation, information, etc., what one does when one does not know what's going on, or when there is a non-zero chance of not knowing what's going on. It is based on focus on the unknown, not the production of mathematical certainties based on weak assumptions or probabilities. Therefore, sometime the "probabilistic veil" can be very opaque computationally, and misplaced precision leads to information opacity, fuzziness, irreversibility, chaos, complexity and confusion [23]. Thus, one of the key questions in understanding the quantum-classical transition is what happens to the superposition as you go up that atoms-to-apple scale. Exactly when and how does "both/and" become "either/or" [22]? Decoherence [23] offers a way to understand classicality as emergent from within the quantum formalism, and the classical world no longer sits in opposition to quantum mechanics, but is demanded by it. In previous paper, we presented a modelling and computational solution to system decoherence transition and entropy conundrum by computational information conservation theory (CICT) [23]. In present paper, we focus our attention on the relativistic electrodynamics observer space-time split (STS) case (previous point A) supported by spacetime algebra (STA) representation scheme and the proposal of a pre-spatial arithmetic scheme (previous point B) supported by CICT.

\section{Method}

We need to make immediately the distinction between "Real" and "Reality" by human worldview [24]. Real means "what it is," while Reality is connected to the "learning resistance" in our human experience, according to our rational learning resources and tools. The Real is, by definition, veiled forever, while the Reality is accessible to our understanding and knowledge by continuous learning. Therefore, Reality can be supported by many different formalisms or description systems (formal languages used by rational observers), but they may not use the same information content or to be endowed with the same inference power. In fact, any inertial observer employs symbolic variables to relate invariant physical quantities to build human being rational (formal) representation of Real which 
he/she is experiencing and he/she is immersed within, by a subjective, advanced virtual holographic projection system powered by his/her brain [25]. To gain a better understanding about the STS operational compromise offered by STA, in the following section (2.1) we offer a brief review of spacetime representation systems, first. Then, we present STA fundamental properties (section 2.2). In section 3., we discuss STS. In section 4. we underline representation system (RS) limitation and ambiguities for low-dimensional representation systems. In section 5., a pre-spatial arithmetic scheme (previous point B) supported by CICT is presented. Section 6 arrives to a generalized narrative view of Section 5 fundamental result. Last Section 7 sums up our findings from a top level point of view.

\subsection{A Brief Review of Spacetime Representation Systems (RS)}

As an example with important implications, let us focus our attention on relativistic electrodynamics briefly. In complete accordance with Nicolescu [24], the classical physics is founded on the idea of continuity (Continuum Hypothesis, $\mathrm{CH}$ ), in agreement with the evidence supplied by the sense organs: we cannot pass from one point of the space and of the time to another, without passing through all intermediary points. The idea of continuity is intimately linked to a key concept of the classical physics: the local causality. Every physical phenomenon could be understood by a continuous chain of causes and effects: to every cause at a certain point corresponds an effect to an infinitely near point and to every effect at a certain point corresponds a cause to an infinitely near point. There is no need of any direct action at distance. The modern science is still founded on the idea of a total separation between the observing-subject and the Reality, assumed to be completely independent from the first one [24]. The "objectivity" of classical physics (Science 1.0, [23]) is fundamentally linked to the knowledge of an object moving in the 1-dimensional time and the 3-dimensional space. The central role of the spacetime (ST) in four dimensions was not altered by the two relativity theories of Einstein, restricted and general, that constitute the apogee of classical physics. QM is a marvelous extension of the classical mechanics. According to Planck's discovery, the energy has a discontinuous, discrete structure at global level. The discontinuity means that between two points there is nothing (Discreteness Hypothesis, DH), no objects, no atoms, no molecules, no particles, just nothing. And even the word "nothing" is too much. A physical quantity has, in QM, several possible values associated with given probabilities of occurrence. But in a physical measurement we get obviously just one single result. This abolition, via the measurement process, of the plurality of possible values of an observable quantity had an obscure meaning but it already clearly indicated the existence of a new type of causality [23]. Seven decades after QM was born, the nature of this new type of causality was clarified thanks to a rigorous theoretical result, the Bell's theorem, and also to high precision experiments. A new concept made in this way its entrance in physics: the non-separability [24]. The quantum entities continue to interact, never mind the distance between them. Therefore, a new type of causality appears, called "global causality," that concerns the system of all physical entities, in their ensemble. The quantum entities are at the same time corpuscles and waves or, more precisely, they are neither corpuscles nor waves. The famous uncertainty relations of Heisenberg show without any ambiguity that is impossible to localize a quantum particle into an exact point of the space and an exact point of time. In other words, it is impossible to assign a well-determined trajectory to a quantum particle [24]. The indeterminism, reigning at the quantum scale, is a structural indeterminism, 
fundamental and irreducible. It does not mean neither hazard nor imprecision [24]. We like to show how ST invariant physical quantities can be related to the variables employed by a specific interacting observer with large information loss unavoidably, if specific awareness and care are not used.

Hendrik Lorentz (1853-1928) [26-28] first applied his well-known transformations to moving electromagnetic bodies using the Heaviside formulation. His initial results were corrected and symmetrized by Henri Poincaré (1854-1912) [29],[30] who suggested that the Lorentz transformation could be considered as a geometric rotation of a 4-vector that had an imaginary time coordinate $(c t) i$ scaled by a constant $c$ (corresponding to the speed of light in vacuum). This was the first unification of space and time into a single 4-vector entity of spacetime. The need for this invariant constant $c$ was independently noticed by Albert Einstein (1879-1955) [31], and elevated to a postulate for his celebrated special theory of relativity that removed the need for a background aether. The 4-vector formalism suggested by Poincaré was fully developed by Hermann Minkowski (1864-1909) [32], but Einstein himself argued against this construction [33] until his later work on gravitation made it necessary [34]. In fact, one hundred years ago, in November 1915, Albert Einstein wrote down the famous field equations of General Relativity. General Relativity is the theory that explains all gravitational phenomena we know (falling apples, orbiting planets, escaping galaxies...) and it survived one century of continuous tests of its validity. After 100 years it should be considered by now a classic textbook theory, but General Relativity remains young in spirit: its central idea, the fact that space and time are dynamical and influenced by the presence of matter, is still mind-boggling and difficult to accept as a well-tested fact of life [35]. Its fundamental properties related to the "Science 2.0" paradigm [23] have not yet been completely grasped by many contemporary scientific disciplines and current researchers, so that not all the implications of this big change have been realized hitherto, even less their related, vital applications. The 4-vector formalism of Poincaré and Minkowski was meanwhile developed by Arnold Sommerfeld (1868-1951) [36],[37], who emphasized that the electromagnetic field was not a 4-vector or combination of 4-vectors, but instead was a different type of object entirely that he called a "6-vector." This 6-vector became intrinsically complex due to the imaginary time $(c t) i$; specifically, it had both electric and magnetic components that differed by a factor of $i$ and transformed into one another upon Lorentz boosts. Bernhard Riemann (1826-1866) [38] and Ludwik Silberstein (1872-1948) [39],[40] independently noted this intrinsic complexity while working in the Heaviside formalism, which prompted them to write the total nonrelativistic field as the single complex vector in eq.(1), in agreement with the relativistic 6-vector construction of Sommerfeld [36],[37]. In mathematical physics, in particular electromagnetism, the Riemann-Silberstein vector, named after Bernhard Riemann and Ludwik Silberstein, (or sometimes ambiguously called the "electromagnetic field") is a complex vector that combines the electric field $\vec{E}$ and the magnetic field $\vec{B}$, on a common region of spacetime, as [41]:

$$
\vec{F}=(\vec{E}+\vec{B} c i)
$$

where $c$ is the speed of light. Some authors prefer to multiply the right hand side by an overall constant $\sqrt{\varepsilon_{0}}$ where $\varepsilon_{0}$ is the permittivity of free space (vacuum):

$$
\vec{F}=(\vec{E}+\vec{B} c i) \sqrt{\varepsilon_{0}}
$$


or equivalently, by an algebraic point of view for representation systems (RS), and more precisely, by a computational point of view for description systems (DS) [42]:

$$
\vec{F}=(\vec{E} / c+\vec{B} i) / \sqrt{\mu_{0}}
$$

where $\mu_{0}$ is the parameter that international Standards Organizations call the "magnetic constant," commonly called vacuum permeability, with the "defined" value $4 \pi \times 10^{-7} \mathrm{H}^{-\mathrm{m}^{-1}}$ [43]. The RiemannSilberstein vector is analogous to the electromagnetic tensor $F^{\mu v}$, a 2-vector used in the covariant formulation of classical electromagnetism. In Silberstein's formulation, $i$ was defined as the imaginary unit, and $\vec{F}$ was defined as a complexified 3-dimensional vector field. The value of $\vec{F}$ at an event was a bivector (a "bivector" is the vector part of a biquaternion; the biquaternions are just the complexification of the (real) quaternions). Incidentally, by quantum mechanics point-of-view, the quantum mechanical derivation that produces eq.(3) also produces Maxwell's vacuum equations in an intriguing form that resembles the Dirac equation for massless spin-1 particles [44-50]. Treating the complex vector in eq.(3) as a single-particle wave function in the first-quantized quantum mechanical sense produces consistent results, and suggests that this vector should be a more natural representation for classical e.m. fields as well. Remarkably, performing the standard second-quantization procedure using the complex Riemann-Silberstein wave function in eq.(3) identically reproduces the correctly quantized electromagnetic field [46-48]. Nevertheless, Minkowski [51] dropped the explicit scalar imaginary $i$ attached to the time coordinate in favor of a different definition of the 4-vector dot product that produced the needed factor of "-1" directly. This change in 4-vector notation removed the ad hoc scalar imaginary, but also effectively discouraged the continued development of the 6-vector of Sommerfeld (and the Riemann-Silberstein vector) by making the complex structure of spacetime implicit. In the absence of an explicit complex structure to distinguish the electric and magnetic field components, these components were reassembled into a rank-2 antisymmetric tensor (i.e., a multilinear antisymmetric function taking two vector arguments), whose characteristic components could be arranged into a $4 \times 4$ antisymmetric matrix $\left[F^{\mu v}\right]$. Although the same components are preserved, this tensor formulation is more difficult to directly relate to the 3-vector formalism. Of particular concern for practical applications, the familiar 3-vector cross product introduced by Willard Gibbs (18391903) could no longer be used with the updated Minkowski 4-vector formalism, and its component representation with tensors like $F^{\mu v}$ was less conceptually clear. Without this cross product, laboratory practitioners had reduced physical intuition about the formalism, which hampered derivations and slowed the adoption of the relativistic formulation. In fact, an algebraic solution to this problem in the form of the wedge product had already been derived by Hermann Grassmann (1809-1877) [52] and William Clifford (1845-1879) [53],[54], and had even been adopted by Élie Cartan's (1869-1951) theory of differential forms [55]. Though this solution was partly rediscovered by Edwin Wilson (1879-1964) and Gilbert Lewis (1875-1946) [56], it remained obscure to the physics community at the time. As such, the readily available methods for practical calculations with 4-vectors and tensors were either (a) to work in component-notation like $F^{\mu v}$ (and akin to the original papers by Maxwell), effectively obscuring the implicit algebraic structure of the relativistic theory, or (b) to convert the equations back into the nonrelativistic 3-vector notation and abandon manifest Lorentz covariance. The choice between these two practical alternatives has essentially created a cultural divide in the 
development and understanding of electromagnetism. The approach using nonrelativistic 3-vectors is still dominant in application-oriented fields like optics where physical intuition is required, while the component notation is dominant in theoretical high energy physics and gravitational communities that cannot afford to hide the structural consequences of relativity, though Cartan's differential forms do occasionally make an appearance. Indeed, the formal manipulation of components using indexnotation have essentially become synonymous with tensor analysis. In Figure 1 a brief historical overview of geometroalgebraic and algebrogeometric representation systems evolution in current scientific arena is offered.

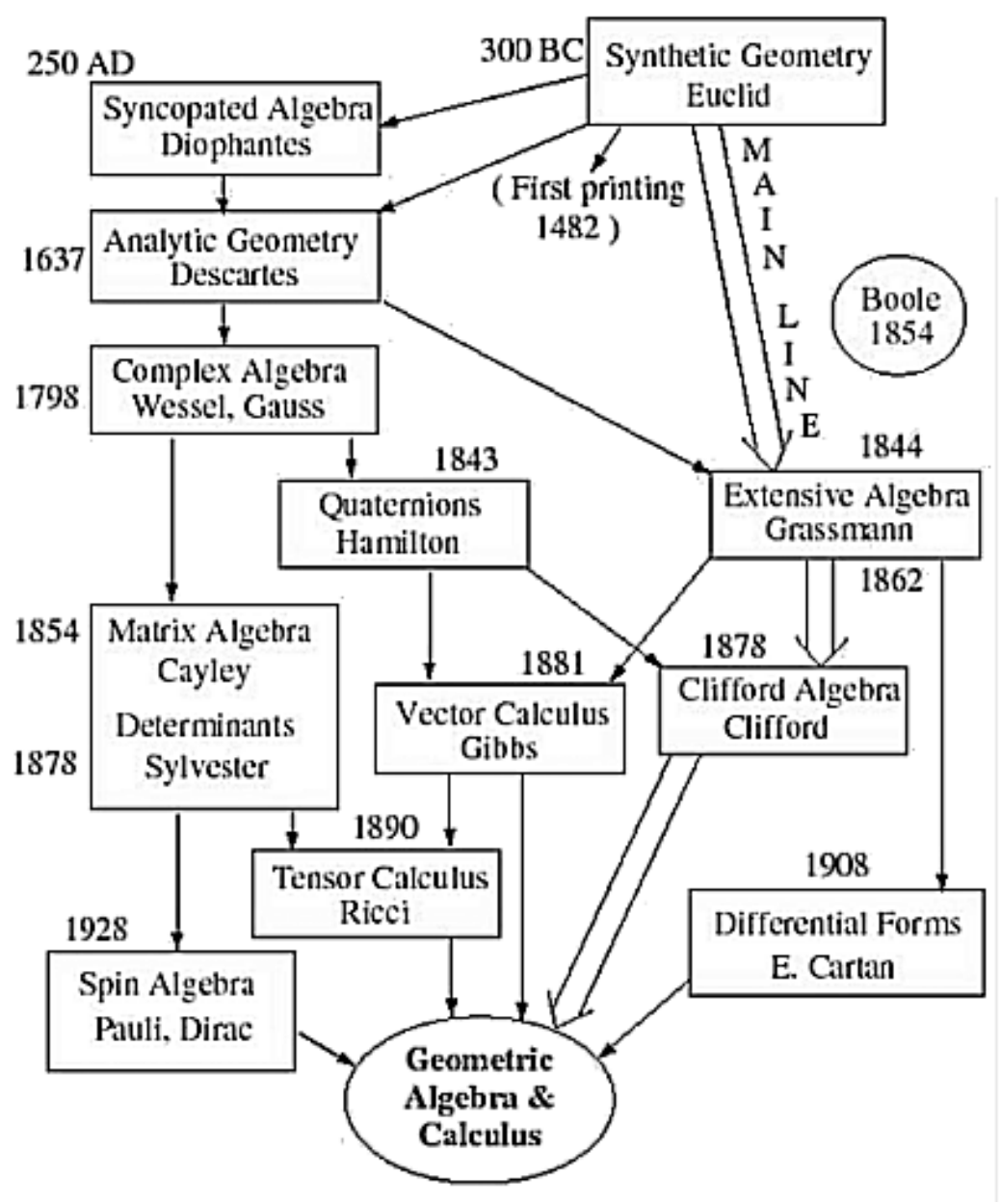

Figure 1. A Brief Historical Overview of Geometroalgebraic and Algebrogeometric Representation Systems evolution (see text) in current scientific arena.

\subsection{STA fundamental properties}

An elegant solution to this growing divide in formalisms was proposed a half-century later by David Hestenes (1933-) [57],[58]. He carefully revisited and further developed [59],[60] the geometric and algebraic work of Grassmann [52] and Clifford [53],[54] (Figure 1). The feature making Clifford algebra (CA) [61],[62] stand out among algebraic systems is the geometric interpretation which can be assigned to multiplication operation. Although the interpretation is not part of the formal algebraic system, it determines how the system is developed and applied, in particular, how it is applied to 
physics, to systems biology, bioengineering, computer science modelling problems and physical system properties representation in general. Unlike the dot and cross products used in standard 2-D and 3-D vector analysis, the Clifford product between vectors is often invertible and not constrained to three dimensions. Unlike the component manipulations used in tensor analysis, spacetime algebra (STA) permits compact and component-free derivations that make the intrinsic geometric significance of physical quantities transparent. This interpretation turns CA into the grammar for a language describing something beyond itself, the Geometric Algebra (GA). The development of GA into a fullblown Geometric Calculus (GC) capable of handling all aspects of differential geometry required a fusion of differential and integral calculus with the concepts of GA in the past years [58],[59]. This task involved a reformulation of manifold theory at the most fundamental level and has been carried out with considerable detail since the 1960s. GA has been used in many physics and engineering applications [63],[64]. The key feature of GA is its ability to express physical equations in a coordinate-free form; the algebra is also extendible to any dimension. When STA is augmented with $\mathrm{GC}$ then it subsumes many disparate mathematical techniques into a single comprehensive formalism, including complex numbers, quaternions, tensors etc. (Figure 1) and provide simple and intuitive geometric interpretations of objects and often operators to construct a full STA. Moreover, for those who are unfamiliar with any of these mathematical techniques, STA provides an encompassing framework that encourages seamless transitions from familiar techniques to unfamiliar ones as the need arises. This modern formalism has the great benefit of preserving and embracing all the existing algebraic approaches in use, but it also extends them in a natural way to allow simple manipulations of relativistic invariant objects with no dimensional limitation.

\section{Space-Time Split (STS)}

Recall that in special relativity [32] one postulates that the scalar time $t$ and vector spatial coordinates $\mathbf{x}$ in a particular inertial reference frame always construct an invariant interval $\left((c t)^{2}-|\mathbf{x}|^{2}\right)$ that does not depend on the reference frame, where $c$ is the speed of light in vacuum. An elegant way of encoding this physical postulate is to combine the scaled time and spatial components into a proper 4-vector composed from combination of one time-like basis vector $\gamma_{0}$ and three orthogonal space-like vectors $\left\{\gamma_{1}, \gamma_{2}, \gamma_{3}\right\}$, with a squared length equal to the invariant interval [33]. The proper notion of length is defined using the relativistic scalar (dot) product between two 4-vectors, which can be understood as a symmetric bilinear function $\eta_{\mu v}$ that takes two vector arguments " $\mu$ " and " $v$ " and returns their scalar shared length. This dot product is known as the Minkowski metric [51]. Note that there is a sign-ambiguity in the spacetime interval, so one can seemingly choose either $(+,-,-,-)$ or $(-,+,+,+)$ for the metric signature, the latter being the initial choice of Poincaré. However, this choice is not completely arbitrary from an algebraic standpoint: it produces geometrically distinct spacetime algebras [64]. We choose the signature here that will produce the STA that correctly contains the relative Euclidean 3-space as a proper subalgebra. Since there are one positive sign and three negative signs in this dot product, we say that it has a mixed signature $(+,-,-,-)$ and denote the vector space of 4-vectors as $\mathcal{M}_{1,3}$ to make this signature explicit. So, all physical vector quantities in special relativity are postulated to be 4 -vectors in $\mathcal{M}_{1,3}$ that satisfy the Minkowski metric. These vectors are geometric quantities that do not depend on the choice of reference frame, so they shall be called proper 
relativistic objects in what follows. Importantly, the Minkowski metric is qualitatively different from the standard Euclidean dot product since it does not produce a positive length. Indeed, the mixture of positive and negative signs can make the length of a vector positive, negative, or zero, which produces three qualitatively different classes of vectors. We call these classes of vectors timelike, spacelike, and lightlike, respectively. Therefore, STA is built up from combinations of one time-like basis vector $\gamma_{0}$ and three orthogonal space-like vectors $\left\{\gamma_{1}, \gamma_{2}, \gamma_{3}\right\}$, under the multiplication rule [58]:

$$
\gamma_{\mu} \gamma_{\nu}+\gamma_{\nu} \gamma_{\mu}=2 \eta_{\mu \nu}
$$

where $\eta_{\mu v}$ is the Minkowski metric with signature (+ - - -). Physically speaking, STA is a complete and natural algebraic language for compactly describing physical quantities that satisfy the postulates of special relativity. Mathematically speaking, it is the largest associative algebra that can be constructed with the vector space of spacetime equipped with the Minkowski metric. It is an orthogonal Clifford algebra [61], which is a powerful tool that enables manifestly frame-independent and coordinate free manipulations of geometrically significant objects. We start from classical approach, taking into consideration a generic electromagnetic field $\vec{F}$, described by Riemann-Silberstein vector in eq.(1). The field $\vec{F}$ is defined as a complexified 3-dimensional vector field. The value of $\vec{F}$ at an event is a bivector according to GA [56]. The field bivector $\vec{F}$ is the same for all observers; there is no question about how it transforms under a change of reference system. However, it is easily related to a representation of coupled electric and magnetic fields in a given inertial system. GA can show how spacetime invariant physical quantities can be related to the variables employed by an inertial observer quite easily. An inertial system is determined by a single unit time like vector $\gamma_{0}$, which can be regarded as tangent to the worldline of an observer at rest in the system. According to STA this vector determines a "split" of ST into Space and Time, in other words a projection from 4-dimensional space into (3+1)-dimensional space, with a chosen reference frame by means of the following two operations [58]:

1- a collapse of the chosen time axis, yielding a 3-D space spanned by bivectors;

2- a projection of the 4D space onto the chosen time axis, yielding a 1D space of scalars;

which are most simply expressed by the following equation [58]:

$$
x \gamma_{0}=t+\mathbf{x}
$$

where $t=x \gamma_{0}$ (dot product) and $\mathbf{x}=x \wedge \gamma_{0}$ (wedge product). We denote the vectors in $\boldsymbol{R}^{\mathbf{3}}$ with boldface type to distinguish them from vectors in $R^{1,3}$. Let $R^{p, q}$ denote a vector space of dimension $n=p+q$ over the reals consisting of a $p$-dimensional subspace of vectors with positive signature orthogonal to a $q$-dimensional subspace of vectors with negative signature [58]. We argue that it is exactly at this point that "both/and" becomes "either/or" representation by usual Science 1.0 approach. In other words, quantum system representation is mapped to classical system additive representation with loss of overall system fundamental coherence information (the field $\vec{F}$ information phase connecting time component to space component coherently into their primitive spacetime continuum, at their original gauge level). In fact, according to $\mathrm{CA}$ traditional approach, this is just an example of a simple projective transformation [58]. It is only a linear mapping of each spacetime point $x$ into a scalar $t$ 
designating a time (divergent or convergent component) and a vector $\mathbf{x}$ designating a position (convergent or divergent component). Classically, the position vectors for all spacetime points compose a 3-dimensional Euclidean vector space $\boldsymbol{R}^{\mathbf{3}}$. In this context, "orthogonality" of vectors means a vanishing inner product. Let $R_{p, q}$ denote the CA generated by the vectors of $R^{p, q}$ and let $R_{p, q}^{k}$ be the $\left(\begin{array}{l}n \\ k\end{array}\right)$-dimensional subspace of $k$-vectors in $R_{p, q}$. Elements of the 1-dimensional subspace $R_{p, q}^{n}$ are called "pseudoscalars" [57] of $R^{p, q}$ or $R_{p, q}$. An arbitrary element of $R_{p, q}$ is called a "multivector" or Clifford number [57]. The primary reference-frame-independent objects of special relativity (i.e., scalars, 4vectors, bivectors/anti-symmetric rank-2 tensors, pseudo-4-vectors, and pseudoscalars) are unified as distinct grades of a single object known as a "multivector." An element of grade $k$ geometrically corresponds to an oriented surface of dimension $k$ (e.g., 4-vectors are line segments, while bivectors are oriented plane segments) [57]. For the important case of a vector space with Euclidean (positive) signature, it is convenient to introduce the abbreviations $R^{n} \equiv R^{n, \circ}, R_{n} \equiv R_{n, 0}$, and $R_{n}^{k} \equiv R_{n, 0}^{k}$. The equation $\mathbf{x}=x \wedge \gamma_{0}$ tells us that a vector in $\boldsymbol{R}^{\mathbf{3}}$ is actually a "bivector" [58] in $R^{2}{ }_{1,3}$. In fact, $\boldsymbol{R}^{\mathbf{3}}$ consists of the set of all bivectors in $R^{2}{ }_{1,3}$ which have the vector $\gamma_{0}$ as a factor. Algebraically, this can be characterized as the set of all bivectors in $R_{1,3}^{2}$ which anticommute with $\gamma_{0}$. This determines a unique mapping of the electromagnetic bivector $\vec{F}$ into the geometric algebra $R_{3}$ of the given inertial system [58]. The STS of $\vec{F}$ by $\gamma_{0}$ is obtained by decomposing $\vec{F}$ into a part [58]:

$$
\vec{E}=1 / 2\left(\vec{F}-\gamma_{0} \vec{F} \gamma_{0}\right)
$$

which anticommutes with $\gamma_{0}$ and a part:

$$
\vec{B} \subset I=1 / 2\left(\vec{F}+\gamma_{0} \vec{F} \gamma_{0}\right)
$$

which commutes with $\gamma_{0}$, so we have:

$$
\vec{F}=(\vec{E}+\vec{B} c I)
$$

where $I=\gamma_{0} \gamma_{1} \gamma_{2} \gamma_{3}=\gamma_{0123}$ is the unit pseudoscalar. By iteratively appending all objects generated by the wedge product (3.4) to the initial vector space $\mathcal{M}_{1,3}$, we construct the full spacetime algebra $\mathrm{C} \ell_{1,3}$. This notation indicates that the spacetime algebra is a Clifford algebra generated from the metric signature $(+,-,-,-)$. Importantly, all components in this Clifford algebra are purely real.

\section{Representation System (RS) Limitation and Ambiguities}

From the beginning with the discovery of complex numbers by Caspar Wessel (1745-1818) in 1797, independently rediscovered by Jean-Robert Argand (1768-1822) in 1806 and Carl Friedrich Gauss (1777-1855) in 1831, the unit imaginary $i$, as in eq.(1), was interpreted as the generator of rotations in a 2-D space [57]. Moreover, development of the subject was stimulated by physical interpretations of complex functions as electric fields, magnetic fields or velocity fields of fluid flow in a physical plane. Despite the great richness and power of complex function theory, it suffers from a defect in design which makes it difficult to see how the theory should be generalized beyond two dimensions. Fortunately, the defect is easily repaired. The defect is an ambiguity in geometrical representation [57]. In fact, throughout the subject, complex numbers play two distinctly different geometrical roles which are not differentiated by the mathematical formalism. On the one hand, a 
complex number may represent a "point in a plane;" on the other hand it may represent a "rotationdilation." Moreover, the representation of points has the defect of singling out a preferred direction (the real axis) with no geometrical or physical significance in applications. The defect can be removed by regarding the "plane" of complex numbers as the even subalgebra $R_{2}^{+}$of the geometric algebra $R_{2}$ for the "real plane" $R^{2}$. Then we can write [57]:

$$
R_{2}=R^{2}+R_{2}^{+}
$$

exhibiting the entire algebra $R_{2}$ as the sum of the real and complex planes. The crucial points of this construction is that the unit imaginary $i$ is now to be interpreted as a "bivector" [58] the unit pseudoscalar for the real plane $R^{2}$, but it is also the generator of rotations in the real plane. For it is easily proved that any vector in $R^{2}$ multiplied by it is rotated by $90^{\circ}$. Parenthetically, we could have achieved the same result by using the geometric algebra $R_{0,2}$ instead of $R_{2}=R_{2,0}$. But then the vectors would have negative square, which might be a drawback or an advantage, depending on what you want to do with it. The advantage is that $R_{0,2}$ can be identified with the quaternions and the even subalgebra of $R 3$. A linear mapping of the real plane $\{\mathbf{x}\}=R^{2}$ onto the complex plane $\{z\}=R^{+}$is defined by the equation $z=\mathbf{x e}_{1}$, where $\mathbf{e}_{1}$ is a fixed unit vector specifying the direction which is mapped into the real axis. By the way, the same kind of linear mapping of vectors into the even subalgebra is of great importance in all CA, because it defines a linear relation between CAs of different dimension. Hestenes calls it a "projective mapping" [58],[65], because it provides an algebraic formulation for the basic idea of introducing homogeneous coordinates in projective geometry. We can use this projective mapping between the real and complex plane to map functions of a complex variable to functions on the real plane where there is no preferred real axis. The GA generated by $\boldsymbol{R}^{\mathbf{3}}$ is identical with the even subalgebra of $R_{1,3}$, so we can write [57]:

$$
R_{3}=R^{+}{ }_{1,3}
$$

Moreover, eq.(8) determines a split of the bivectors in $R_{1,3}$ into vectors and bivectors of $R_{3}$, as expressed by writing [57]:

$$
R^{2}{ }_{1,3}=R^{1}{ }_{3}+R^{2}{ }_{3}
$$

This split is not unique, however, as it depends on the choice of the vector $\gamma_{0}$. STA is the Hestenes' name for the Clifford algebra $C \ell_{1,3}(\mathrm{R})$, or equivalently the geometric algebra $\mathrm{G}_{4} \equiv \mathrm{G}\left(\mathcal{M}_{1,3}\right)$. A complete discussion of STSs is given in references [58] and [62]. Of course, $\vec{E}$ and $\vec{B}$ in eq.(8) are just the classic coupled electric and magnetic fields in the $\gamma_{0}$-system, and the additive split of $\vec{F}$ into electric and magnetic fields will be different for different inertial systems. As we briefly indicated, the embedding of Clifford algebras $\mathrm{C} \ell_{3,0} \subset \mathrm{C} \ell_{1,3}$ motivated our choice of metric signature for spacetime to be $(+,-,-,-)$. In fact, this embedding is part of a larger sequence of Clifford algebra embeddings starting from $C \ell_{0,0} \subset \mathrm{Cl}_{0,1} \subset \mathrm{Cl}_{0,2} \subset \mathrm{Cl}_{3,0} \subset \mathrm{C \ell}_{1,3} \subset \mathrm{C} \ell_{4,1} \subset \mathrm{C \ell}_{2,4}$ which corresponds to, respectively, the real numbers, complex numbers (Schrödinger spinors), quaternions (Hamilton spinors), relative 3space (Pauli spinors), spacetime/electromagnetism (Maxwell spinors), relativistic electron (Dirac spinors), and conformal space (Penrose twistors) [66]. Here, we like to focus your attention to the fact that the classic vector space $\mathcal{M}_{1,3}$ does not contain the complete physical picture implied by special relativity, since it must be augmented by quantities like $F^{\mu v}$ to obtain the complete picture of special 
relativity in a systematic and principled way. Indeed, standard treatments of electromagnetism, involving relative 3 -vectors, use both the symmetric dot product and the antisymmetric vector cross product to properly discuss the physical implications of the theory. The classic vector space $\mathcal{M}_{1,3}$ only specifies the relativistic version of the dot product in the form of the Minkowski metric. As you can see from previous presentation in Section 3., without introducing the proper relativistic notion of the wedge product (proper generalization of the vector cross product to relativistic 4-vectors) the physical picture of spacetime is incomplete. Mathematically, the introduction by STA of a product on a vector space creates an algebra and the wedge product is precisely Grassmann's exterior wedge product, adopted by Cartan to define differential forms [52]. The wedge product enlarges the mathematical space in order to properly accommodate quantities like the electromagnetic field tensor $F^{\mu v}$. Evidently, the intrinsic complex structure inherent to the geometry of spacetime has deep and perhaps underappreciated consequences for even our classical field theories. The complex vector from eq.(8) will appear naturally as a relative 3-space expansion of a bivector field, which is a proper geometric (and thus frame-independent) object in spacetime. Moreover, this bivector field will have a crucial fundamental difference from eq.(1): the scalar imaginary $i$ will be replaced by an algebraic unit pseudoscalar $I$ (also satisfying $I^{2}=-1$ ) that is intrinsically meaningful to and required by the geometric structure of spacetime. This replacement makes the complex form of eq.(1) reference-frame independent, which is impossible when the mathematical representation is restricted to the usual scalar imaginary $i$ only. The electromagnetic field is an irreducibly complex object with an intrinsic phase. This phase necessarily involves the intrinsic pseudoscalar (unit 4-volume) $I$ of spacetime, and is intimately related to the appearance of electromagnetic waves and circular polarizations, with no need for any ad hoc addition of a complex scalar field. As a matter of fact, this replacement of the scalar $i$ with $I$ has to be systematic throughout the electromagnetic theory, where it appears in electromagnetic waves, elliptical polarization, the normal variables for canonical field mode quantization, and the dual symmetric internal rotations of the vacuum field, to arrive to full representation information conservation.

\section{CICT Pre-Spatial Arithmetic Scheme}

To conserve the full spacetime information content, we need to avoid the loss of overall system fundamental information (the field $\vec{F}$ information phase connecting time component to space component coherently into their primitive spacetime continuum, at their original gauge level), which is manifestly an ill-posed problem by classic approach (Science 1.0). In fact, through the e.m. potential, the system gets a chance to communicate with other systems. Notice that all e.m. interactions occur in a two-level way; the potential keeps the interacting particles phase-correlated whereas the combination of its space-time derivatives, named e.m. field, accounts for the forces involved. The lower level, the potential, becomes physically observable only when the phase $\Phi$ of the system assumes a precise value in a specific gauge field. The structure of electrodynamics makes possible the presence of a potential also when both electric and magnetic fields are absent, whereas on the contrary fields are always accompanied by potentials. This solution which stems from the mathematical formalism of QFT [9] opens the possibility of tuning the fluctuations of a plurality of systems, producing therefore their cooperative behavior. However, some conditions must be met in order to implement such a possibility. 
Let us, first of all, realize that in quantum physics the existence of gauge fields, such as the e.m. potential, dictated by the physical requirement that the quantum fluctuations of atoms should not be observable directly, prevents the possibility of having isolated bodies. For this reason, the description of a physical system is given in terms of a matter field, which is the space-time distribution of atoms/molecules, coupled to the gauge field with the possible supplement of other fields describing the nonelectromagnetic interactions, such as the chemical forces. According to the principle of complementarity, there is also another representation where the phase assumes a precise value; this representation which focuses on the wave-like features of the system cannot be assumed simultaneously with the particle representation. The relation between these two representations is expressed by the uncertainty relation, similar to the Heisenberg relation between position and momentum:

$$
\Delta N \Delta \Phi \geq 1 / 2
$$

connecting the uncertainty of the number of quanta (particle structure of the system) $\Delta N$ and the uncertainty of the phase (which describes the rhythm of fluctuation of the system) $\Delta \Phi$. Consequently, the two representations we have introduced above correspond to the two extreme cases:

(A) If $\Delta N=0$, the number of quanta is well defined, so that we obtain an atomistic description of the system, but lose the information on its capability to fluctuate, since $\Delta \Phi$ becomes infinite. This choice corresponds to the usual description of objects in terms of the component atoms/molecules;

(B) If $\Delta \Phi=0$, the phase is well defined, so that we obtain a description of the movement of the system, but lose the information on its particle-like features which become undefined since $\Delta N$ becomes infinite. Such a system having a well-defined phase is termed "coherent" in physics.

In the phase representation, the deepest quantum features appear since the system becomes able to oscillate with a well-defined phase only when the number of its components becomes undefined, so that it is an open system and able to couple its own fluctuations to the fluctuations of the surroundings. In other words, such a coherent system, like a biological one, is able to "feel" the environment through the e.m. potential created by its phase dynamics. In conclusion, a coherent system involves two kinds of interaction:

(A) an interaction similar to that considered by Classical Physics, where objects interact by exchanging energy. These exchanges are connected with the appearance of forces measured by their magnitude (modulus) only, in an assumed continuum manifold that may be approached and studied by traditional stochastic and probabilistic tools offered by the large arena of the Geometric Science of Information (GSI). Since energy cannot travel faster than light, this interaction obeys the principle of causality. The missing part of this worldview is usually called "system noise", "background radiation", etc... on cosmic scale by human being (Science 1.0 approach);

(B) an interaction where a common phase arises among different objects because of their coupling to the quantum fluctuations and hence to an e.m. potential. In this case there is no propagation of 
matter and/or energy taking place, and the components of the system "talk" to each other through the modulations of the phase field travelling at the phase velocity, which has no upper limit and can be larger than $c$, the speed of light (QFT approach).

Therefore, to grasp a more reliable representation of reality and to get more effective modeling techniques, researchers and scientists need two intelligently articulated hands: both stochastic and combinatorial approaches synergically articulated by natural coupling (Science 2.0 approach) [63]. As a simple example, first we show how to conserve the vector full information content of rational correspondence at higher representation level, and then we discuss a CICT pre-spatial arithmetic scheme to vanish the probabilistic modeling veil opaqueness.

\subsection{Vanishing the Probabilistic Veil Opaqueness}

According to Hestenes [57], we can take the mathematical concepts of modular magnitude and direction as basic, and introduce the concept of vector as the basic kind of directed number, with an additional, associated, specific phasing relation. Directed numbers are defined implicitly by specifying rules for adding and multiplying vectors. According to CICT vector representation precision point of view [67], directed numbers (modular magnitude and direction) can be related uniquely to long division rational remainder sequences to identify convenient "quantum support field", which subspace inner phased generators can be computed from. CICT new awareness of a discrete HG (hyperbolic geometry) subspace (reciprocal space) of coded heterogeneous hyperbolic structures, underlying the familiar $\boldsymbol{Q}$ Euclidean (direct space) surface representation, shows that any natural number $n$ in $\boldsymbol{N}$ has associated a specific, non-arbitrary extrinsic or external phase relationship that we have to take into account to full conserve overall system information content by computation in Euclidean space. This new awareness can open the way to holographic information geometry (HIG) to recover system lost coherence and to overall system entropy minimization. CICT emerged from the study of the geometrical structure of a discrete manifold of ordered hyperbolic substructures, coded by formal power series, under the criterion of evolutive structural invariance at arbitrary precision. It defines an arbitrary-scaling discrete Riemannian manifold uniquely, under HG metric, that, for arbitrary finite point accuracy level $L$ going to infinity under scale relativity invariance, is isomorphic (even better, homeomorphic) to classic Riemannian manifold (exact solution theoretically). In other words, HG describes a projective relativistic geometry directly hardwired into elementary arithmetic long division remainder sequences, offering many competitive computational advantages over traditional Euclidean approach. This is the new "Science 2.0" fundamental computational rationale behind any arbitrary precision elementary dichotomy structuring, to be compared to usual "Science 1.0" dichotomy by cutting and splitting only (rounding as best case). We refer to solid number (SN) and its quotient and remainder sequence RFD (Representation Fundamental Domain) concepts as in [68]. As a simple example, for $\mathrm{SN}=7.0=D$, to conserve the full information content of rational correspondence at higher level, we realize that we have to take into account not only the usual modulus information, but even the related external or extrinsic RFD periodic precision length information $\mathrm{W}=6$ (numeric period or external phase representation) in this case (i.e. CD1 $=000007$ as base RFD, and yes for 
CICT leading zeros do count [23]!). We can use Euler's formula to establish the usual fundamental relationship between trigonometric functions and the complex exponential function:

$$
e^{i x}=\cos x+i \sin x
$$

where $e$ is the base of the natural logarithm and $i=\sqrt{ }-1$. It establishes the fundamental relationship between the trigonometric functions and the complex exponential function. We obtain:

$$
C Q 1=\frac{1}{7} e^{i \frac{\pi(2 n+1)}{3}}=\frac{1}{7}\left(\cos \left(\frac{2 \pi(n+1)}{6}\right)+i \sin \left(\frac{2 \pi(n+1)}{6}\right)\right)
$$

and

$$
C D 1=\frac{1}{C Q 1}=7 e^{-i \frac{\pi(2 n+1)}{3}}=7\left(\cos \left(-\frac{\pi(2 n-1)}{3}\right)+i \sin \left(-\frac{\pi(2 n-1)}{3}\right)\right)=7\left(\frac{1}{2}-i \frac{\sqrt{3}}{2}\right) \text { p.v. }
$$

for $n=1,2,3, \ldots$ in $N$, where p.v. means principal value. CICT shows that any natural number $D$ in $N$ has associated a specific, non-arbitrary external or exterior phase relationship [69] that we must take into account to full conserve its information content by computation in Euclidean space [70]. The interested reader will have already guessed the relationship of our result to de Moivre number or root of unity (i.e. any complex number that gives 1.0 when raised to some integer power of $n$. In this way, we can exploit Rational numbers $\boldsymbol{Q}$ full information content to get effective and stronger solutions to current system modelling problems. Now, to go through the probabilistic modelling veil opaqueness, we have to take advantage from the CICT pre-spatial arithmetic scheme, offered by RFD rational remainder sequence. For $\mathrm{SN}=7.0=D$, it is immediate to see that for long division 1 by 7 , we have:

$$
\begin{array}{lll}
\mathrm{L}_{1}=1, & \mathrm{Q}_{1}=1 / 10, & \mathrm{R}_{1}=3 / 10^{1} ; \\
\mathrm{L}_{2}=2, & \mathrm{Q}_{2}=14 / 10^{2}, & \mathrm{R}_{2}=02 / 10^{2} ; \\
\mathrm{L}_{3}=3, & \mathrm{Q}_{3}=142 / 10^{3}, & \mathrm{R}_{3}=006 / 10^{3} ; \\
\mathrm{L}_{4}=4, & \mathrm{Q}_{4}=1428 / 10^{4}, & \mathrm{R}_{4}=0004 / 10^{4} ; \\
\mathrm{L}_{5}=5, & \mathrm{Q}_{5}=14285 / 10^{5}, & \mathrm{R}_{5}=00005 / 10^{5} ; \\
\mathrm{L}_{6}=6, & \mathrm{Q}_{6}=142857 / 10^{6}, & \mathrm{R}_{6}=000001 / 10^{6} ; \\
\mathrm{L}_{7}=7, & \mathrm{Q}_{7}=1428571 / 10^{7}, & \mathrm{R}_{7}=0000003 / 10^{7}
\end{array}
$$

and the associated RFD for $\mathrm{Q}_{k}$ and $\mathrm{R}_{\mathrm{k}}$ is just given by $\mathrm{Lx}_{\mathrm{x}}=6$, and RFD period is $\mathrm{T}_{\mathrm{p}}=6$, in this case. Therefore, SN full information content is captured by a six-digit word length $\mathrm{RFD}_{6}$, for $\mathrm{Q}_{6}$ and the same for R6. Eqs. (16) sum up the first stages of elementary long division of 1 by 7 . But from eqs.(16) it is straightforward to note that:

$$
\frac{1}{7}=\frac{1}{10} \sum_{k=0}^{\infty}\left(\frac{3}{10}\right)^{k}=\frac{14}{10^{2}} \sum_{k=0}^{\infty}\left(\frac{02}{10^{2}}\right)^{k}=\frac{142}{10^{3}} \sum_{k=0}^{\infty}\left(\frac{006}{10^{3}}\right)^{k}=\ldots \ldots \ldots \ldots . . .
$$

In other words, the Remainder $\mathrm{R}_{\mathrm{L}}$, at any computation evolutive stage, is the fixed multiplicative ratio of a formal power series associated to optimal decimal representations of $1 / 7$, at increasing arbitrary accuracy levels. Thus $1 / 7$ associated information content can be lossless compressed down to minimal $\mathrm{M}_{\mathrm{QR}}=(1+1)$ digits in total, in this case. As a matter of fact, any word couple $<\mathrm{Q}_{\mathrm{L}}, \mathrm{R}_{\mathrm{L}}>$ can be thought to be equivalent to and can represent a different real measurement instrument class, defined 
by RFD word length $\mathrm{Lx}_{\mathrm{x}}$. By realizing that the remainder $\mathrm{R}_{1}$ is the fixed multiplicative ratio of a formal power series, the computation of $3^{\mathrm{n}}(\bmod 7)$ for $\mathrm{n}=1,2,3, \ldots$, till its exponential closure, gives the "Fundamental Cyclic Remainder Sequence" (FCRS):

$$
\mathrm{R}_{1}=3, \mathrm{R}_{2}=2, \mathrm{R}_{3}=6, \mathrm{R}_{4}=4, \mathrm{R}_{5}=5, \mathrm{R}_{6}=1
$$

from which the "Fundamental Cyclic Quotient Sequence" (FCQS) can be readily regenerated by $7 * \mathrm{R}_{\mathrm{n}}$ $(\bmod 10)$ :

$$
\mathrm{Q}_{1}=1, \mathrm{Q}_{2}=4, \mathrm{Q}_{3}=2, \mathrm{Q}_{4}=8, \mathrm{Q}_{5}=5, \mathrm{Q}_{6}=7 \text {. }
$$

So, quotient and remainder information can always be regenerated LTR (Left-To-Right) anew by remainder information only, but not vice-versa. Thanks to the above properties, the division algorithm can become free from trial and error like in Finite Segment P-adic representation systems, but with no usually associated coding burden [68],[71]. Analogous reasoning shows that quotient and remainder information can always be regenerated RTL (Right-To-Left) anew by remainder information only, but not vice-versa for divergent sequences. In this way, we arrive to CICT fundamental relationship that ties together numeric body information of divergent and convergent monotonic power series in any base (in this case decimal, with no loss of generality), with $D$ ending by digit 9 :

$$
\frac{1}{D}=\sum_{k=0}^{\infty} \frac{1}{10^{W}}\left(\frac{\bar{D}}{10^{W}}\right)^{k} \Rightarrow \ldots \Leftarrow \operatorname{Div}\left(\frac{1}{D}\right)=\sum_{k=0}^{\infty}(D+1)^{k}
$$

where $\bar{D}$ is the additive $10^{W}$ complement of $D$, i.e. $\bar{D}=\left(10^{W}-D\right), W$ is the word representation precision length of the denominator $D$ and "Div" means "Divergence of". Further generalizations related to $D$ ending by digit 1 or 3 or 7 are straightforward [67]. Reversing a convergent sequence into a divergent one and vice-versa is the fundamental property to reach information conservation, i.e. information reversibility (Figure 2).

\section{$1 /(\mathrm{N}-1) \longleftrightarrow 1 / \mathrm{N} \longmapsto 1 /(\mathrm{N}+1)$}

Upscale Contiguity Operator Downscale Contiguity Operator

$$
\sum_{k=1}^{\infty}\left(\frac{1}{N}\right)^{k}=\frac{1}{N-1}, k=1,2,3, \ldots \in N \quad \sum_{k=0}^{\infty}(-1)^{k}\left(\frac{1}{N}\right)^{(k+1)}=\frac{1}{N+1}, k=0,1,2,3, \ldots \in N
$$

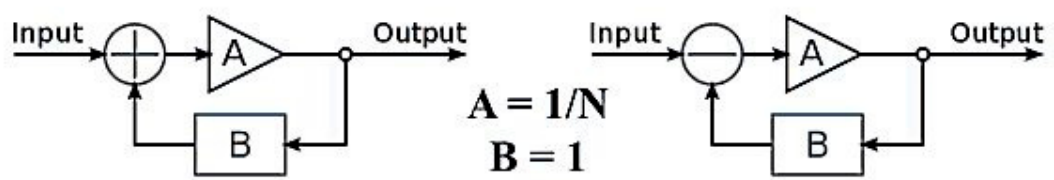

Figure 2. Egyptian fractions $\boldsymbol{Q}$ subset as the discrete continuum of connected rationals (with no loss of generality) with two basic contiguity operators for LTR (downscale) and RTL (upscale) sequencing (full information reversibility). 
CICT results have been presented in term in term of classic or formal power series to show the close relationship to classic and modern control theory approaches for causal continuous-time and discrete-time linear systems, allowing information folding and unfolding, according to specific modeling needs, with no information dissipation. Increasing the subspace representation accuracy, the total number of allowed convergent paths, as monotonic power series, for instance (as allowed subspace paths), increases accordingly till maximum machine word length and beyond; like discrete quantum paths denser and denser to one another, towards a never ending "blending quantum continuum," called "quantum mixture" by the traditional Top-Down (TD) statistic perspective for composite multi-scale system (Science 1.0 approach). While differentiable trajectories found in standard mathematical physics are automatically scale invariant, it is the main insight of the CICT theory that also certain non-differentiable paths (resultant paths, emerging from lower scales combined quantum trajectory interactions, which explicitly depend on the scale and accuracy of the observer) can be scale invariant. Scale related, coherent precision correspondence leads to transparency, ordering, reversibility, cosmos, simplicity, clarity, and to algorithmic quantum incomputability on real macroscopic machines [23]. According to CICT, the full information content of any symbolic representation emerges out of the capturing of two fundamental coupled components: the linear one (unfolded) and the nonlinear one (folded). Referring to the transdisciplinary concept interpretation presented in [25], we see that for full information conservation any transdisciplinary concept mustb emerge out of at least two pair of fundamental coupled parts, even by a narrative point of view. As an example, for STS, we can arrive to better operative understanding of usual term, with added possibility of information conservation, as shown by "The Four Quadrants of The Space-Time Split" (Figure 3). In other words by CICT, if we want to capture the full information content of any linear elementary symbolic representation, we need a quadratic support space at least. Of course, we can apply our dichotomizing process in a recursive way to achieve any precision we like.

\section{Generalized Narrative View}

From previous sections, we have seen that ST invariant physical quantities can be related to the variables employed by a specific interacting observer to get an interpretation of the world which human being is immersed within. In that passage information can be lost or dissipated to unaware interactor. In fact, we have seen that original "spacetime" ST (a transdisciplinary concept) gets split into two separated additive subcomponents "space" and "time." That operational splitting can represent an advantage by a formal (rational) representation point-of-view (POV) (i.e. easiness of representation), but the usual major drawback is an original information precision loss, if we are unaware of it, or we are unable to compensate for, not taking into consideration the folding and unfolding properties offered by computational information conservation theory (CICT) "OperRational" representation [68]. For instance, we can arrive to better operative understanding of usual terms, with added possibility of information conservation as shown in "The Four Quadrants of The Space-Time Split" (Figure 3) by a narrative POV (point-of-view). Here, the term "Timeline" (first quadrant, top right) is considered the combination of a major linear time representation framed by folded minor space representation. The term "Overview" (second quadrant, top left) is interpreted as the combined representation of major linear space and major linear time representations, with minor complementary 
folded time and space components. The term "Snapshot" (third quadrant, bottom left) can be assumed as the combination of a major linear space representation framed by minor folded time representation. The forth quadrant (bottom right) represents the combination of major folded space and time components, framed by the combination of minor linear space and time components. In can be interpreted as a simple (bidimensional), but realistic representation of usual information flow as experienced by living organism.

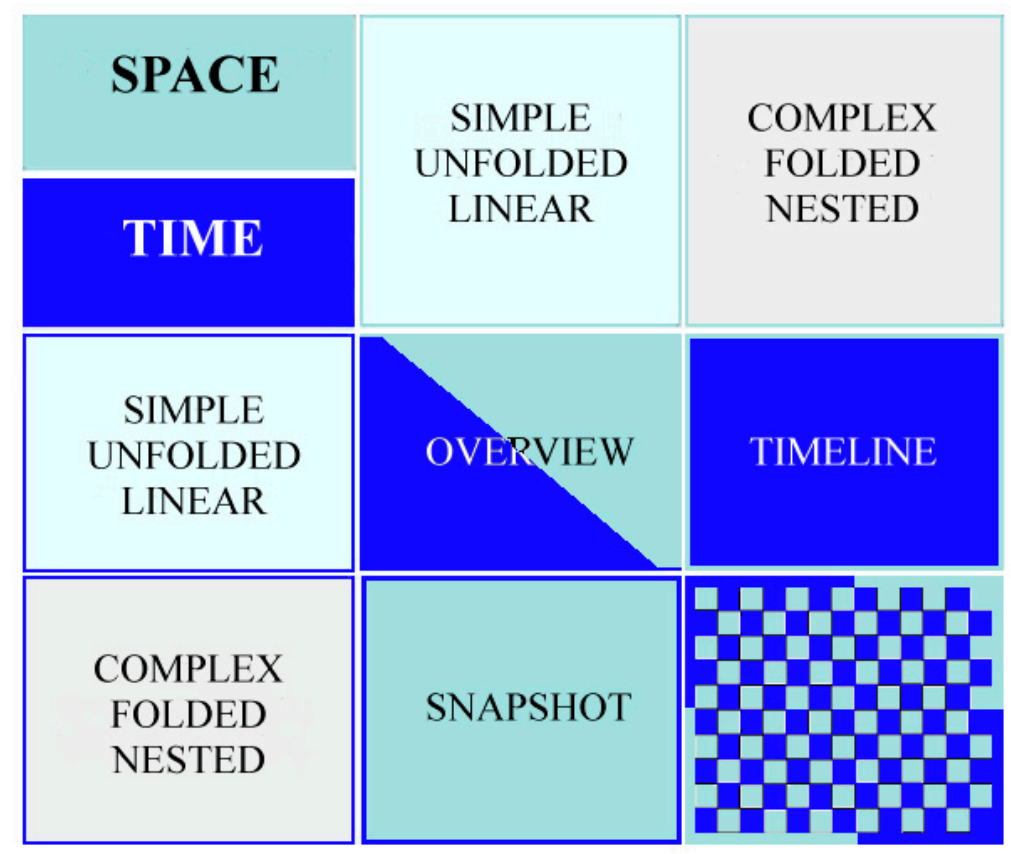

Figure 3. The Four Quadrants of The Space-Time Split (STS) (see text).

The current living generation is experiencing a transition from history to hyperhistory. Advanced information societies are more and more heavily dependent on ICTs (Information and Communication Technologies) for theirs normal functioning and growth. Processing power will increase, while becoming cheaper and cheaper. The amount of data will reach unthinkable quantities. And the value of our networked resources will grow almost vertically. However, our storage capacity (space) and the speed of our communications (time) are lagging behind. Hyperhistory is a new era in human development, but it does not transcend the spatio-temporal constraints that have always regulated our life on this planet. The question to be addressed next is: "given all the variables we know, what sort of hyperhistorical environment are we building for ourselves and for future generations?" The short answer is: the Infosphere. The final result is CICT new awareness of a discrete HG (hyperbolic geometry) subspace (reciprocal space) of coded heterogeneous hyperbolic structures, underlying the familiar $\boldsymbol{Q}$ Euclidean (direct space) surface representation. It can open the way to holographic information geometry (HIG). CICT can help us to develop strategies to gather much more reliable experimental information from single experimentation and to conserve overall system information. In this way, coherent representation precision leads to information conservation and clarity. Specifically, advanced mathematical modeling, advanced wellbeing applications (AWA), high reliability organization (HRO), mission critical project (MCP) system, very low technological risk (VLTR) and crisis management (CM) system will be highly benefitted mostly by these new techniques. 


\section{Conclusion}

The process of the emergence of coherent structures out of a crowd of independent component particles has been investigated in the last decades and is presently quite well understood [72],[73]. The presence of this electromagnetic field $\vec{F}$ has received experimental corroboration by the discovery of the so-called "Lamb shift," named after the Nobel prize winner Lamb [74]. He discovered as far back as in 1947 that the energy level of the electron orbiting around the proton in the hydrogen atom is slightly shifted (about one part per million) with respect to the value estimated when assuming that no electromagnetic field is present. Further corroboration for the existence of vacuum fluctuations is provided by the Casimir effect [75]. Therefore a weak electromagnetic field is always present, just the one arising from the vacuum quantum fluctuations. According to QFT, the gauge invariance in quantum physics becomes the natural partner of the phase invariance to produce the representation and description of our world. Quantum fluctuations give rise to electromagnetic potentials which spread the phase fluctuations beyond the system at the phase velocity. This gives an intrinsic nonlocalizability to the system and prevents a direct observation of quantum fluctuations. Through the electromagnetic potential, the system gets a chance to communicate with other systems and subsystems [10]. Reversing a convergent sequence into a divergent one and vice-versa is the fundamental property to reach information conservation, i.e. information reversibility. Eventually, CICT OECS have strong connection even to classic DFT algorithmic structure for discrete data, Number-Theoretic Transform (NTT), Laplace and Mellin Transforms [69]. As a matter of fact, to grasp a more reliable representation of reality, researchers and scientists need two intelligently articulated hands; both stochastic and combinatorial approach synergistically articulated by natural coupling; let's say we need a fresh "Science 2.0" approach [76]. Therefore, CICT Coherent precision correspondence allows us to go through the probabilistic veil arriving to transparency, ordering, reversibility, cosmos, simplicity and clarity, by a simple arithmetic scheme strongly coupled to GA and GC representations. This paper is a relevant contribution towards an effective and convenient "Science 2.0" universal framework to develop more effective physics modeling and simulation for innovative application and beyond.

\section{Conflicts of Interest}

The author declares no conflict of interest.

\section{References}

1. Di Sia, P. Classical and quantum transport processes in nano-bio-structures: a new theoretical model and applications, PhD Thesis. Verona University, Italy, 2011.

2. Di Sia, P. Present and Future of Nanotechnologies: Peculiarities, Phenomenology, Theoretical Modelling. Perspectives. Reviews in Theoretical Science, 2014, 2, 146.

3. Di Sia, P. Interesting Details about Diffusion of Nanoparticles for Diagnosis and Treatment in Medicine by a new analytical theoretical Model. Journal of Nanotechnology in Diagnosis and Treatment, 2014, 2, 6 . 
4. Di Sia, P. Relativistic nano-transport and artificial neural networks: details by a new analytical model. International Journal of Artificial Intelligence and Mechatronics (IJAIM), 2014, 3, 96100.

5. Andrews, A.M.; Weiss, P.S. Nano in the Brain: Nano-Neuroscience, ACS Nano, 2012, 6, 84638464.

6. Azevedo, F.A.C.; Carvalho, L.R.B.; Grinberg, L.T.; Farfel, J.M.; Ferretti, R.E.L.; Leite, R.E.P.; Herculano-Houzel, S. Equal Numbers of Neuronal and Nonneuronal Cells Make the Human Brain an Isometrically Scaled-Up Primate Brain. Journal of Comparative Neurology, 2009, 513, 532541.

7. Alivisatos, A.P. Chun, M.; Church, G.M.; Greenspan, R.J.; Roukes, M.L.; Chun Yuste, R. The Brain Activity Map Project and the Challenge of Functional Connectomics. Neuron, 2012, 74, 970-974.

8. Alivisatos, A.P.; Andrews, A.M.; Boyden, E.S.; Chun, M.; Church, G.M.; Deisseroth, K.; Zhuang, $\mathrm{X}$. Nanotools for neuroscience and brain activity mapping. ACS Nano, 2013, 7, 1850-66.

9. Fiorini, R.A. Arbitrary multi-scale (AMS) systems biology and biomedical engineering effective modeling and simulation. Journal of biology and biomedical systems, WSEAS Press, 2015, submitted.

10. Fiorini,R.A. A natural framework for arbitrary multi-scale computer science and systems biology efficient computational modeling. In Proceedings of the $6^{\text {th }}$ International Conference on Applied Informatics and Computing Theory (AICT 2015), 27-29 June 2015, Salerno, Italy; Recent Advances in Computer Engineering Series n.31, June 2015, WSEAS Press, pp.31-40.

11. Mishra, A. K. (Ed.) Nanomedicine for Drug Delivery and Therapeutics; Wiley: USA, 2013.

12. Resconi, G. Geometry of Knowledge for Intelligent Systems; Springer International, Vol.407 of Studies on Computational Intelligence, 2012.

13. Nugent, M.A.; Molter, T. W. AHaH Computing-From Metastable Switches to Attractors to Machine Learning. PLOS ONE, 2014. doi:10.1371/journal.pone.0085175.

14. Resconi, G.; Licata, I. Computation by Intention and Electronic Image of the Brain. Journal of Applied \& Computational Mathematics, 2015, 4, 232-244.

15. Licata, I. Effective Physical Processes and Active Information in Quantum Computing. Quantum Biosystems, 2007, 1, 51-65.

16. Licata, I. Beyond Turing: Hypercomputation and Quantum Morphogenesis. Asia Pacific Mathematics Newsletter, 2012, 2, 20-24.

17. MacLennan, B. Natural computation and non-Turing models of computation. Theoretical Computer Science, 2004, 317, 115-145.

18. MacLennan, B. Molecular Coordination of Hierarchical Self-Assembly, Technical Report UT-CS10-662, 2010. Available online: http://web.eecs.utk.edu/ mclennan/papers/MCHSA-TR.pdf .

19. Ghirardi, G. Sneaking a Look at God's Cards: Unraveling the Mysteries of Quantum Mechanics; Malsbary G (translator), Princeton University Press: Princeton, NJ, 2007.

20. Heisenberg, W. Die physikalischen Prinzipien der Quantentheorie; S. Hirzel: Leipzig, 1941.

21. Chiatti L.; Licata I. Relativity with respect to measurement: collapse and quantum events from Fock to Cramer. Systems, 2014, 2, 576-589.

22. Ball, P. Physics: Quantum all the way, Nature, 2008, 453, 22-25. 
23. Fiorini, R.A. The Entropy Conundrum: A Solution Proposal. In Proceedings of the 1st Int. Electron. Conf. Entropy Appl., 3-21 November 2014; Sciforum Electronic Conference Series, 2015, 1, a011; doi:10.3390/ecea-1-a011. Available online: http://sciforum.net/conference/ecea1/paper/2649 (accessed on 13 October 2015).

24. Nicolescu, B. Heisenberg and the Levels of Reality, European Journal of Science and Theology, 2006, 2, 9-19.

25. De Giacomo, P.; Fiorini, R.A. Creativity Mind (Preview); ebook, Amazon, 2015.

26. Lorentz, H.A. Simplified theory of electrical and optical phenomena in moving systems. Proc. Roy. Neth. Acad. Arts Sci., 1899, 1, 427-442.

27. Lorentz, H.A. Weiterbildung der Maxwellshen Theorie. Elektronentheorie. Ency. Math. Wiss., 1904, 5, 145-288.

28. Lorentz, H.A. Electromagnetic phenomena in a system moving with any velocity smaller than that of light. Proc. Roy. Neth. Acad. Arts Sci., 1904, 6, 809-831.

29. Poincaré, H. Sur la dynamique de l'électron. C. R. Acad. Sci., 1905, 140, 1504-1508.

30. Poincaré, H. Sur la dynamique de l'électron. Rend. Circ. Mat. Pal., 1906, 21, 129-176.

31. Einstein, A. Zur elektrodynamik bewegter Körper. Ann. Phys., 1905, 322, 891-921.

32. Minkowski, H. Das Relativitätsprinzip. Ann. Phys., 1907, 352, 927-938.

33. Einstein, A. Über die elektromagnetischen Grundgleichungen für bewegte Körper. Ann. Phys., 1908, 331, 532-540.

34. Einstein, A. Relativität und gravitation. Erwiderung auf eine Bemerkung von M. Abraham. Ann. Phys., 1912, 38, 1059-1064.

35. Einstein, A. Die Feldgleichungen der Gravitation. Sitzungsberichte der Preussischen Akademie der Wissenschaften zu Berlin, 1915, 844-847.

36. Sommerfeld, A. Zur Relativitätztheorie I: Vierdimensionale Vektoralgebra. Ann. Phys., 1910, 337, 749-764.

37. Sommerfeld, A. Zur Relativitätztheorie II: Vierdimensionale. Ann. Phys., 1910, 337, 765-776.

38. Weber, H. Die Partiellen Differential-Gleichungen Der Mathematischen Physik: Nach Riemann's Vorlesungen Bearbeitet; Friedrich Vieweg und Sohn: Braunschweig, 1901.

39. Silberstein, L. Elektromagnetische Grundgleichungen in bivectorieller Behandlung. Ann. Phys., 1907, 327, 579.

40. Silberstein,L. Nachtrag zur Abhandlung über 'Elektromagnetische Grundgleichungen in bivectorieller Behandlung'. Ann. Phys., 1907, 329, 783.

41. Aste, A. Complex representation theory of the electromagnetic field. Journal of Geometry and Symmetry in Physics, 2012, 28, 47-58.

42. Fiorini, R.A. Sistemi di Supporto Attivo; CUSL: Milano, 1994.

43. The NIST Reference on Constants, Units and Uncertainty. Unit of electric current (ampere), 2015. Available online: http://physics.nist.gov/cuu/Units/ampere.html.

44. Stepanovsky, Y.P. From Maxwell equation to Berry's phase and sonoluminescence: Problems of theory of electromagnetic and other Massless fields. Electromagnetic Phenomena 1, 1998, 180 218.

45. Kobe,D.H. A relativistic Schrödinger-like equation for a photon and its second quantization. Found. Phys., 1999, 19, 1203-1231. 
46. Smith, B.J.; Raymer, M.G. Photon wave functions, wave-packet quantization of light, and coherence theory. New J. Phys., 2007, 9(11), 414.

47. Dragoman, D. Photon states from propagating complex electromagnetic fields. J. Opt. Soc Am., 2007, B24, 922-927.

48. Tamburini, F.; Vicino, D. Photon wave function: A covariant formulation and equivalence with QED. Phys. Rev. A, 2008, 78, 052116.

49. Thidé, B. Electromagnetic Field Theory; Dover Publications: New York, NY, 2014.

50. Thidé, B. The physics of angular momentum radio, 2015. Available online: http://arxiv.org/pdf/1410.4268.

51. Minkowski, H. Raum und Zeit. Phys. Zeit., 1908, 10, 75-88.

52. Grassmann, H. Die Lineale Ausdehnungslehre — Ein neuer Zweig der Mathematik; Verlag von Otto Wigand: Leipzig, 1844.

53. Clifford, W.K. Applications of Grassmann's Extensive Algebra. Am. J. Math., 1878, 1, 350-358.

54. Clifford, W.K. Elements of Dynamic: An Introduction to the Study of Motion and rest in Solid and Fluid Bodies; MacMillan and Co.: London, UK, 1878.

55. Cartan, E. Sur certaines expressions différentielles et le probème de Pfaff. Ann. Sci. l'École Norm. Sup., 1899, 16, 239-332.

56. Wilson, E.B.; Lewis, G.N. The space-time manifold of relativity. The non-Euclidean geometry of mechanics and electromagnetics. Proc. Amer. Acad. Arts Sci., 1912, 48, 387-507.

57. Hestenes, D. A Unified language for Mathematics and Physics. In J.S.R. Chisholm; A.K. Commons (eds.) Clifford Algebras and their Applications in Mathematical Physics. Reidel: Dordrecht/Boston, 1986; pp.1-23.

58. Hestenes, D. Space-Time Algebra; Gordon and Breach: New York, N.Y., 1966.

59. Hestenes, D.; Sobczyk, G. Clifford Algebra to Geometric Calculus; Reidel: Dordrecht/Boston, MA, USA, 1984.

60. Hestenes, D. New Foundations for Classical Mechanics; Reidel Publ. Co.: Dordrecht/Boston, MA, USA, 1985.

61. Hestenes, D. Oersted medal lecture 2002: Reforming the mathematical language of physics. Amer. J. Phys., 2003, 71, 104-121.

62. Hestenes, D. Spacetime physics with geometric algebra. Amer. J. Phys., 2003, 71, 1-24.

63. Hestenes, D. Gauge theory gravity with geometric calculus. Found. Phys., 2005, 35, 903-970.

64. Doran, C.; Lasenby, A. Geometric Algebra for Physicists. Cambridge University Press: Cambridge, UK, 2007.

65. Crumeyrolle, A. Orthogonal and Symplectic Clifford Algebras; Kluwer Academic Publishers: Dordrecht, 1990.

66. .Dressel, J.; Bliokh, K.Y., Nori, F. Spacetime algebra as a powerful tool for electromagnetism. Physics Reports, 2015, 589, 1-71.

67. Fiorini, R.A. Computerized Tomography Noise Reduction by CICT Optimized Exponential Cyclic Sequences (OECS) Co-domain. Fundamenta Informaticae, 2015, 141, 115-134.

68. Fiorini, R.A.; Laguteta, G. Discrete Tomography Data Footprint Reduction by Information Conservation. Fundamenta Informaticae, 2013, 125, 261-272. 
69. Fiorini, R.A. How Random is Your Tomographic Noise? A Number Theoretic Transform (NTT) Approach. Fundamenta Infomaticae, 2014, 135, 135-170.

70. Fiorini, R.A. Computational Information Conservation Theory: An Introduction. In 2014 Proceedings of the 8th International Conference on Applied Mathematics, Simulation, Modelling (ASM '14), 22-24 November 2014, Florence, Italy; N.E. Mastorakis, M. Demiralp, N. Mukhopadhyay, F. Mainardi, eds., Mathematics and Computers in Science and Engineering Series, No.34, WSEAS Press, pp.385-394.

71. Young, D.M.; Gregory, R.T. A Survey of Numerical Mathematics; Addison Wesley: Reading, MA, USA, Vol.I and II, 1973.

72. Preparata, G., QED Coherence in Matter; World Scientific: Singapore, 1995.

73. Del Giudice, E.; Vitiello G. Role of the electromagnetic field in the formation of domains in the process of symmetry breaking phase transitions. Physical Review A, 2006, 74, Article ID 022105.

74. Lamb, W.E.; Retherford R.C. Fine structure of the hydrogen atom by a microwave method. Physical Review, 1947, 72, 241-243.

75. Casimir, H.B.G. On the attraction between two perfectly conducting plates. Proceedings of the Koninklijke Nederlandse Akademie VanWetenschappen B, 1948, 51, 793-796.

76. Fiorini, R.A. GA and CICT for Stronger Arbitrary Multi-Scale Biomedical and Bioengineering Solutions. In Early Proceedings of the AGACSE 2015 Conference, July 29-31, Barcelona, Spain, pp.153-162.

(C) 2015 by the authors; licensee MDPI, Basel, Switzerland. This article is an open access article distributed under the terms and conditions of the Creative Commons Attribution license (http://creativecommons.org/licenses/by/3.0/). 